\title{
Fistula between three main cerebral arteries and a large occipital vein
}

\author{
J. BRET AND Z. KUNC \\ From the Neurosurgical Clinic of the Charles University, Prague, Czechoslovakia
}

The literature contains few references to direct communications between cerebral arteries and veins. Dandy (1945) was the first to describe the passage of a branch from the middle cerebral artery into a vein leading into the Sylvian group. Jaeger and Forbes (1946) reported direct communications between the posterior cerebral and posterior choroid arteries and the basilar vein, together with multiple anastomoses with the great vein of Galen. Haberland (1950) published an account of the accidental finding of a communication between the branches of the posterior cerebral artery and the dilated vein of Rozenthal and proved it microscopically. Bergstrand, Olivecrona, and Tönnis (1936), Röttgen (1937), Sorgo (1938), and Verbiest (1951) reported observations of intradural direct arteriovenous communications. Some cases of vascular malformations of the great vein of Galen may also belong to this category, although they are generally regarded as a separate entity.

The following report describes an abnormality which is unique in our experience, and we have not encountered a similar one in our survey of the literature.

\section{CASE REPORT}

A 26-year-old man was first seen in September 1961. From childhood he had suffered from right-sided headaches, mainly in the temporal region and below the eyebrow, the pain being treated as migraine. As time went on, the headache ceased to trouble him, but he then began to complain of attacks of vertigo, associated with nausea or vomiting, usually lasting about one hour and being so severe that they forced him to go to bed for fear of falling. It was for these attacks that he sought advice.

The neurological examination and funduscopy at that time were normal. Skull films and tomograms revealed a hyperostosis in the left fronto-parietal region, growing from the outer table, with a circular defect, limited sharply by sclerotic bone. In the tangential view there was thickening of the inner table in the base of the hyperostosis. These appearances were suggestive of a meningioma, and angiography was undertaken with this possibility in mind.
LEFT CAROTID ANGIOgram (Fig. 1) This showed a dilatation of the internal carotid artery at its siphon and of the anterior cerebral artery in its first part. The majority of the contrast medium was drawn through the anterior communicating artery to the right anterior cerebral artery, which was dilated to a width of $5 \mathrm{~mm}$ in the early part of its course and $7 \mathrm{~mm}$ further posteriorly. It terminated directly into an enormous occipital vein running to the sagittal sinus. There was poor visualization of the territory supplied by the left anterior and middle cerebral arteries, indicating that most of the blood was going to the fistula.

RIGHT CAROTID ANGIOGRAM (Fig. 2) This also showe dilatation of the internal carotid artery. The beginning of the anterior cerebral artery was not seen. The middieg cerebral artery was greatly enlarged, elongated, meander ing, and terminated in the same anomalous occipita vein as had been seen in the previous angiogram. The vein wound in large loops to the sagittal sinus. The sagittal and both transverse sinuses were also dilatedo As on the left side, the blood supply of the right hemisphere appeared to be deficient, because most of the circulation was going to the fistula.

ANGIOGRAM OF THE LEFT VERTEBRAI. ARTERY (Fig. 3a, b) This showed that the vertebral and basilar arteries were enlarged, elongated, and winding. Almost all the contrast material passed into the right posterior cerebral artery, which was dilated and ran in big curves into the anomalous occipital vein. The cerebellar vessels were poorly visualized.

Total serial angiography confirmed these circulatory anomalies. The patient refused surgical treatment, as he did not consider that his complaints were troublesome enough at this time. He was readmitted on 2 February 1964 because the headaches had recurred, and because he had had several attacks of transient weakness of the right hand, and on two occasions loss of consciousness. The neurological state remained normal.

At operation on 25 February 1964 a right occipitoparietal craniotomy was performed. On the surface of the brain an anomalous vein, as large as a thumb, ran from the occipital to the parietal lobe, forming a large varix just before its termination in the sagittal sinus. N The arachnoid was thickened over the whole of this region. From all directions large arteries entered the vein $\tilde{O}$ so that a thrill could be felt on palpation. When all $\underset{\omega}{\sim}$ these arteries had been ligated, the tension in the vein 


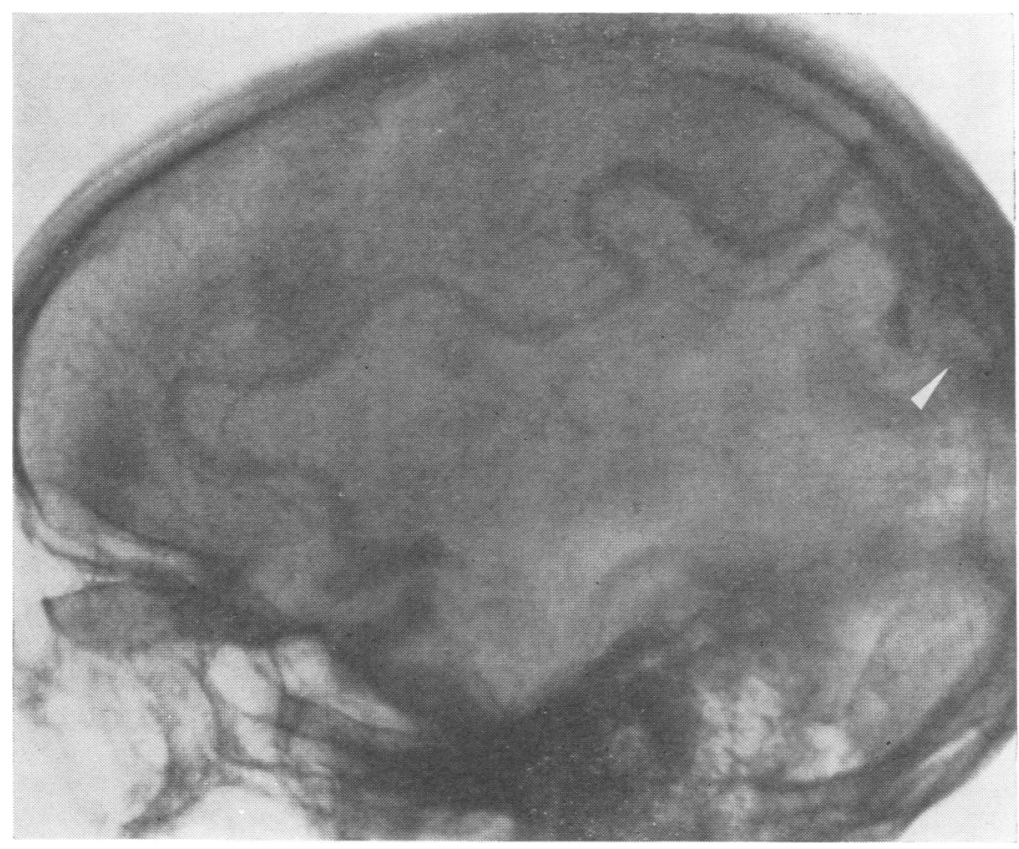

FIG. 1. Before operation.

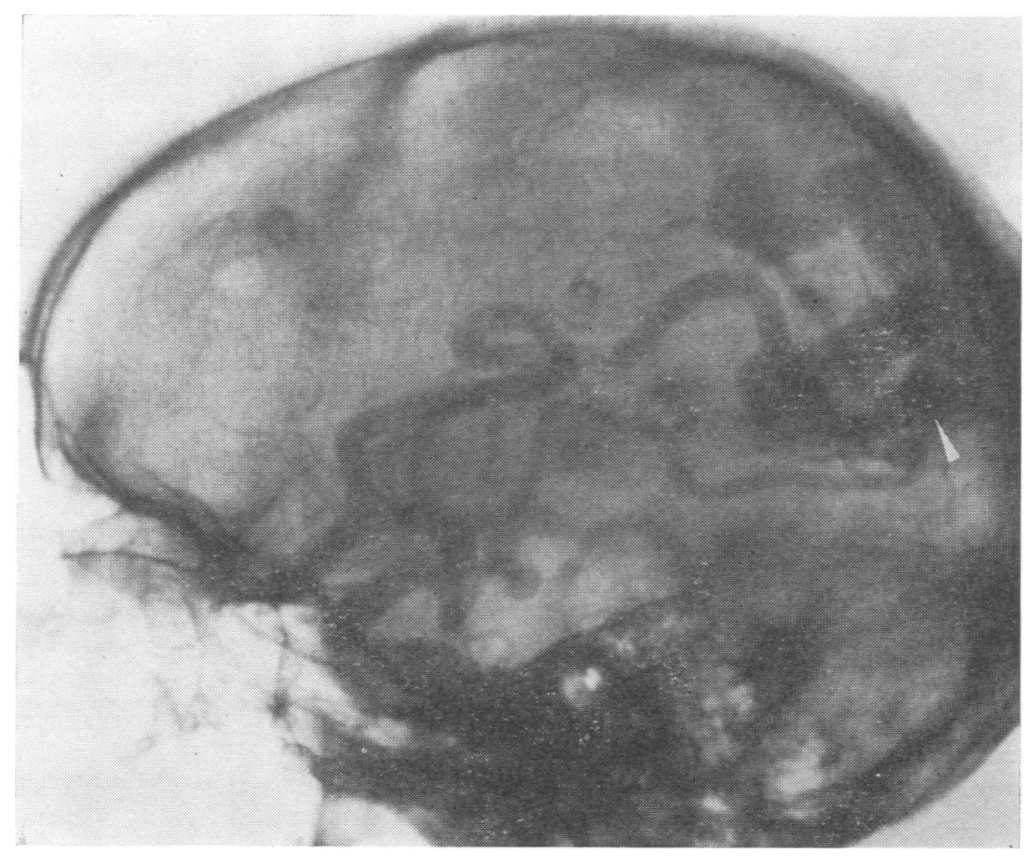

FIG. 2. Before operation. 


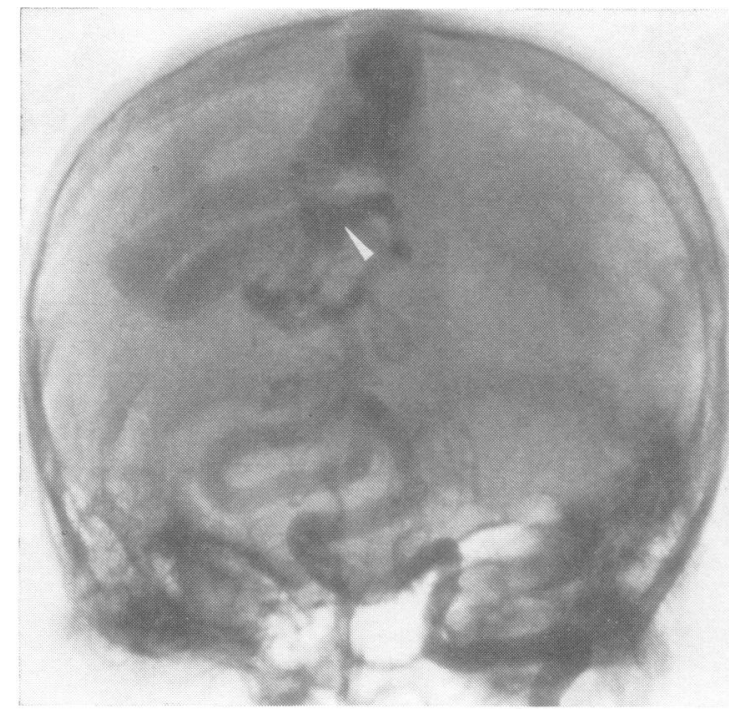

FIG. 3a

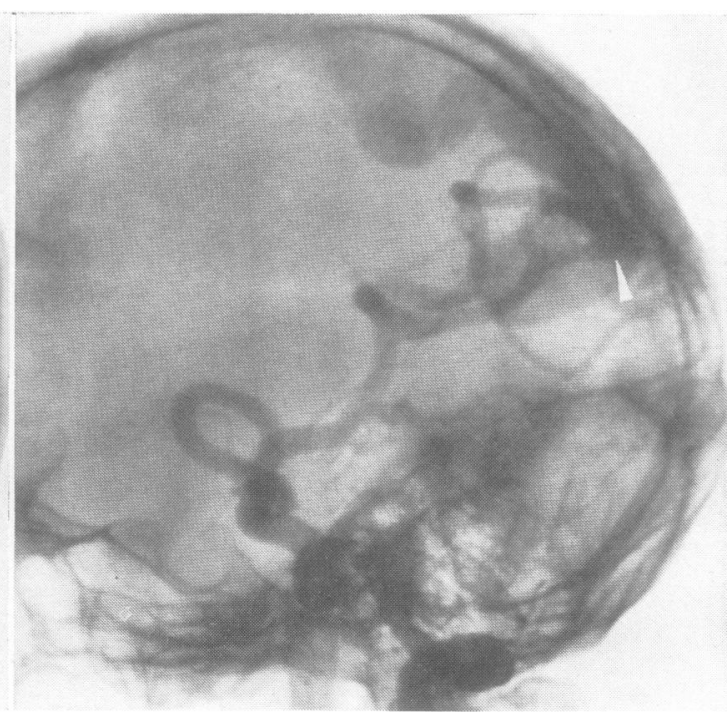

FIG. $3 b$

FIG. 3 Before operation.

greatly decreased and the thrill disappeared. Then the gigantic vein with a part of the brain was removed. The procedure was extremely difficult, as the walls of the vessels were very fragile and, in spite of careful dissection, often tore with violent bleeding, serious blood loss, and drop of the arterial pressure. The patient received a blood transfusion of $4,100 \mathrm{ml}$. The procedure took four hours.

For some days after the operation the patient was somnolent and confused and had a left-sided hemiparesis. This complication cleared up rapidly, but a superficial wound infection required antibiotic therapy for several days. He was discharged in good mental and physical condition. Control angiography was carried out on 1 April 1964 (Fig. 4a, b, c, d). The cerebral circulation in both hemispheres now appeared to be virtually normal. All of the main cerebral arteries on the right side had diminished in size and the arteriovenous communications were no longer visible. Both of the carotid arteries were smaller and irregularly narrowed, but there was an aneurysmal dilatation of the origin of the posterior communicating artery on the left side.

COMMENT The vascular anomaly described above would explain the chronic headache of which the patient had complained since his childhood. All three main cerebral arteries on the right side terminated in an occipitoparietal vein which emptied into the sagittal sinus. All these vessels were enormously dilated, and the fistulous communication between them led to serious deprivation of the blood supply to the whole brain. The radical removal of the malformation led to a complete relief of symptoms.

\section{DISCUSSION}

This vascular anomaly is unique in its form, an in the literature no similar observation could be found. It is the nearest to the one described by Dandy (1945), but in comparison is more com ${ }^{+}$ plicated and larger. In Haberland's (1950) case the arteriovenous communication was microscopic. In other cases described in the literature the lesion has been situated in the dura mater and the arterial communications were mostly from the meningeal arteries. The malformation which we have described is more primitive than in the typical arteriovenous malformations. Between the feeding arteries and draining vein there was no system of transit vascular channels, and so the arterial blood stream ran directly into the enormously dilated cerebral vein.

This arteriovenous fistula is the result of a developmental defect of the capillary system in the early stage of embryonic development of the cerebral vascular system, as in arteriovenous malformations. It is possible that embryonic vessels, destined to be arteries and veins, may cross in such a way that they are separated only by two layers of endothelial cells and that this barrier gradually disappears under the influence of the arterial pulse.

It is probable that the abnormality, present at birth and enlarging steadily thereafter, was responsible for the long-standing headaches. Progressive enlargement of the contributing vessels increased

.


the power of the shunt, and led to a deficit in the cerebral and cerebellar blood supply, witnessed by vertigo, transient hemianopia, and attacks of loss of consciousness. Haberland's findings suggest that arteriovenous communications, at first only microscopic, open successively during life. In our case, the normal development of the brain, as indicated by intact mentality and the absence of neurological signs, suggests that the shunt, as seen at the time of angiography and operation, may have been present at birth, but enlarged progressively throughout life.

The indication for surgery was clear, not only because of severe headache but also because of the prospect of the cerebral circulation becoming more embarrassed, leading to the grave mental changes which are known to occur in large arteriovenous malformations.

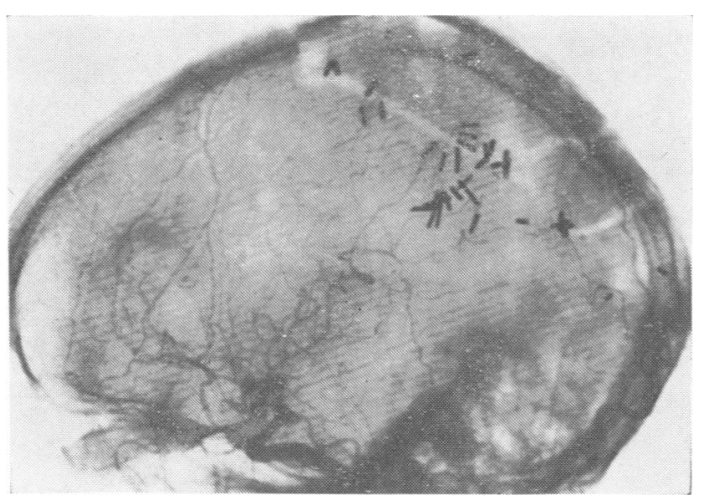

FIG. 4a

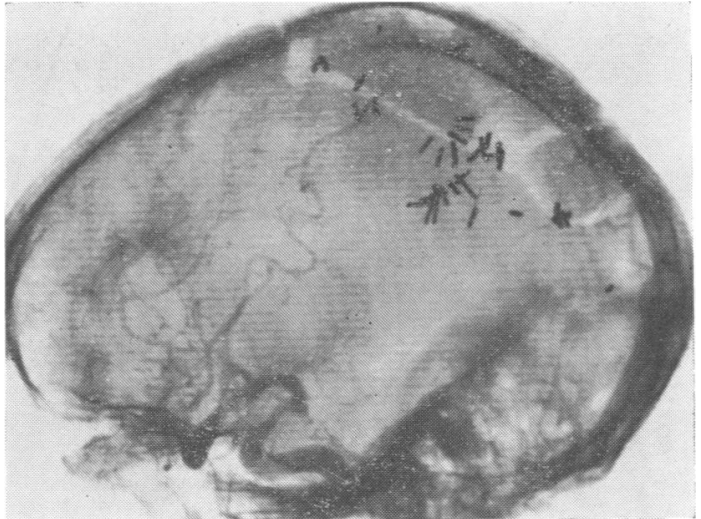

FIG. $4 b$

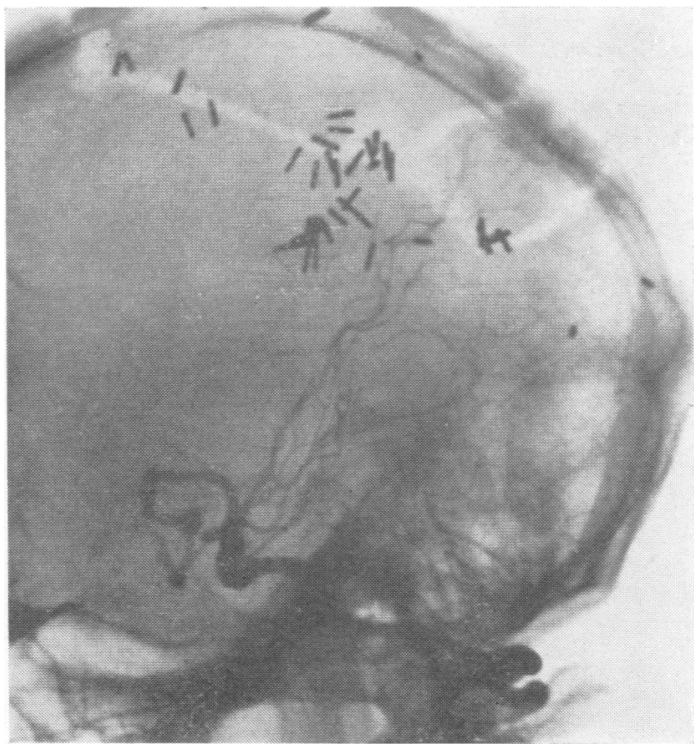

FIG. 4d

FIG. 4 After operation. 


\section{SUMMARY}

A case of unique multiple arteriovenous fistulae between all three main cerebral arteries-anterior, middle, and posterior arteries-and a cortical occipito-parietal vein, is reported. The malformation disturbed the cerebral circulation and caused severe headaches, vertigo, transient hemianopia, and attacks of loss of consciousness. Surgical ablation of the fistula relieved these symptoms and restored the circulation to normal. A possible explanation of the abnormality is offered.

\section{REFERENCES}

Bergstrand, H., Olivecrona, H., and Tönnis, W. (1936). Gefässmissbildungen und Gefässgeschwulste des Gehirns. Thieme: Leipzig.
Dandy, W. E. (1945). Surgery of the brain, in Lewis' Practice of $Z$ Surgery, Vol. 12. Edited by W. Walters. W. F. Prior: Hagerstown, Maryland.

Haberland, C. (1950). Arteriovenous anastomosis on the base of the brain. Mschr. Psychiat. Neurol., 119, 199-206.

Jaeger, R., and Forbes, R. P. (1946). Bilateral congenital arteriovenous communications of the cerebral vessels. Arch. Neurol. Psychiat. (Chic.), 55, 591-599.

Röttgen, P. (1937). Weitere Erfarungen an kongenitalen arteriovenösen Aneurysmen des Schädelinnern. Zbl. Neurochir.,.. 2, 18-34.

Sorgo, W. (1938). Weitere Mitteilungen über Klinik und Histologie des kongenitalen arteriovenösen Aneurysmas des Gehirns. Zbl. Neurochir., 3, 64-87.

Verbiest, M. H. (1951). L'anévrisme artérioveineux intradural. Rev. Neurol., 85, 189-199. 